\title{
Prognostic nomogram to predict overall survival for patients with perihilar cholangiocarcinoma: A population-based study of SEER database
}

\section{Chendong Wang}

Nantong University Medical School

Huanyu Gong

Jiangsu University School of Medicine

Zhiyuan Zhang

Nantong University Medical School

Danzhou Fang

Nantong University Medical School

Huiqun Wu ( $\nabla$ wuhuiqun@ntu.edu.cn )

Research article

Keywords: perihilar cholangiocarcinoma, nomogram, overall survival, prognosis, SEER

Posted Date: July 7th, 2020

DOI: https://doi.org/10.21203/rs.3.rs-40400/v1

License: (1) (i) This work is licensed under a Creative Commons Attribution 4.0 International License.

Read Full License 


\section{Abstract}

Background Perihilar cholangiocarcinoma (pCCA) is a highly aggressive malignancy with poor prognosis. Accurate prediction is of great significance for patients' survival outcome. The present study aimed to propose a prognostic nomogram for predicting the overall survival (OS) for patients with pCCA. Methods We conducted a retrospective analysis in a total of 940 patients enrolled from the Surveillance, Epidemiology, and End Results (SEER) program and developed a nomogram based on the prognostic factors identified from the cox regression analysis. Concordance index (C-index), risk group stratification and calibration curves were adopted to test the discrimination and calibration ability of the nomogram with bootstrap method. Decision curves were also plotted to evaluate net benefits in clinical use against TNM staging system. Results On the basis of multivariate analysis, five independent prognostic factors including age, summary stage, surgery, chemotherapy, together with radiation were selected and entered into the nomogram model. The $\mathrm{C}$-index of the model was significantly higher than TNM system in the training set $(0.703$ vs $0.572, P<0.001)$, which was also proved in the validation set $(0.718$ vs 0.588 , $\mathrm{p}<0.001)$. The calibration curves for 1-, 2-, and 3-year OS probabilities exhibited good agreements between the nomogram-predicted and the actual observation. Decision curves displayed that the nomogram obtained more net benefits than TNM staging system in clinical context. The OS curves of two distinct risk groups stratified by nomogram-predicted survival outcome illustrated statistical difference. Conclusions We established and validated an easy-to-use prognostic nomogram, which can provide more accurate individualized prediction and assistance in decision making for pCCA patients.

\section{Background}

Cholangiocarcinoma (CCA), also named as bile duct cancer, a relatively rare tumor, is the most common biliary tract malignancy and second most common primary liver malignancy [1]. According to different anatomical locations [2], they are classified into intrahepatic cholangiocarcinoma (iCCA), perihilar cholangiocarcinoma (pCCA), and distal cholangiocarcinoma (dCCA). The pCCA, located between the second-degree hepatic ducts and the insertion of cystic duct into the common bile duct [3], also recognized as Klatskin tumor, is the most common subtype, with the proportion of approximately $50 \%$ of cholangiocarcinomas [4]. The prognosis of Klatskin tumor is dismal with $10 \%$ of 5 -year survival rates [5]. It is generally believed that surgical treatment of surgery and liver transplantation remains the mainstay of potentially curative treatment. However, only about one in five patients are candidates for such therapies due to late-stage diseases or metastasis at the time of presentation [6]. Unfortunately, with such a low resection rate, most patients (76\%) will still recur after resection [7], which emphasizes the need for better adjuvant strategies. Some experts held the view that adjuvant therapy can extend the lifespans of patients receiving surgical resection, especially margin-positive resection [8]. For inoperable patients, palliative management with chemotherapy, radiation or chemoradiation also might be life-extending options [4]. Moreover, evidences disclosed that patients could benefit from some novel treatment options like intervention therapy, targeted therapy or immunotherapy $[9,10]$. Currently, the broadly used staging system for perihilar cholangiocarcinoma include Bismuth-Corlette [11, 12] and Memorial Sloan-Kettering 
Cancer Center (MSKCC) staging systems [13], but there are frequently applied to determine the operative methods or predict the resectability and the likelihood of metastasis. Besides, the American Joint Committee on Cancer (AJCC) TNM system and the Mayo Clinic systems are capable of classifying patients into different prognostic stages based on nonoperative information at the diagnosis. However, these previous staging systems only contain a restricted number of prognosis-associated factors, with insufficient considerations on other significant variables, like age, gender, tumor differentiation, and therapeutic strategies. Thus, their roles in accurate prognosis prediction are finite [14-16]. In the era of personalized cancer treatment, an accurate individualized evaluation is critical for personal specific treatment or care options. For the above reasons, the more precise model for predicting survival for patients with pCCA is urgently required.

Nomogram, as a simple graphical visualization of statistical model, is capable of providing individual patient with accurate survival information [17], and it has been proved to be favorable for a range of cancer types [18-22]. Such a prognostic tool has the potential to become an alternative and even a novel standard in cancer prognosis evaluation. With respect to the nomogram established for PCCA, to our knowledge, the model constructed from real world study which combines demographic variants, surgery, chemotherapy, and radiation treatment is still lack. Hence, the current study aimed at the derivation and validation of an effective prognostic nomogram for pCCA patients utilizing the data from the Surveillance, Epidemiology, and End Results (SEER) program.

\section{Methods}

\section{Patients and study design}

The SEER program collects and publishes the cancer occurrence and survival data in the United States, accounting for about $26 \%$ of the population [23]. In this retrospective study, baseline and clinical data of pCCA patients were obtained through the SEER ${ }^{*}$ Stat version 8.3.6 software (Surveillance Research Program, NCl, Bethesda, MD). The inclusion criteria were as follow: (1) Cases diagnosed between 2004 and 2015; (2) Cases with the primary site code of liver, intraheptic duct, and other biliary; (3) Cases diagnosed with the definite histological type of Klatskin tumor (8162/3, based on the third edition of International Classification of Diseases for Oncology [ICD-0-3]). The exclusion criteria included: (1) Patients with unknown demographic information, such as age at diagnosis, gender or race. (2) Patients with unknown survival time information; (3) Patients with unknown diagnostic confirmation or only diagnosed via a death certificate or autopsy; (4) Patients with indefinite treatment information including surgery and radiation. Through patient identification, 940 eligible cases were enrolled to this study. For further analysis, the whole cohort was randomly split into a training set $(n=658)$ and a validation set $(n=$ 282) according to the ratio of 7:3 using 'sample' function in R software. The training set was employed to establish the predictive nomogram, while the validation set for external validation. Ethical approval and informed consent were unnecessary for our study because SEER dataset is publicly available and all patient data are de-identified. We have also signed data use agreement and achieved permission to access the database. 


\section{Variables extraction}

Baseline and clinicopathological characteristics were gathered from the SEER database, including age, race, gender, marital status, grade, T stage (AJCC, 6th ed.), N stage (AJCC, 6th ed.), M stage (AJCC, 6th ed.), SEER summary stage, surgery, radiation, chemotherapy, survival time and vital status. Age at diagnosis was categorized into three subgroups (less than 68 years old, aged between 69 and 84 years old and 85 years or older group). The terms of "Surg Prim Site" and "Reason no cancer-directed surgery" could help identify patients with the receipt of surgery. Surgery and radiation therapy were defined as receiving relevant treatment or not. Chemotherapy was classified as receiving or not/unknown. The endpoint was overall survival (OS), the definition of which is the length of time from diagnosis to allcause deaths or the last follow-up.

\section{Statistical analysis}

Continuous variables were transformed to categorical variables by virtue of cut-off value derived from Xtile software (Version 3.6.1, Yale University, New Haven, CT, USA). Categorical data were compared by Chisquare test or Fisher's exact test. Cox proportional hazards regression model was employed in the univariate and multivariate analysis. Hazard ratio (HR) and relevant $95 \%$ confidence interval $(\mathrm{Cl})$ were calculated. Prognostic predictors were determined by multivariate analysis and a nomogram was formulated. Performance of the constructed nomogram was assessed via Concordance index (C-index) and calibration plots $[24,25]$. Bootstraps with 1,000 resamples were used in validation. Calibration plots represented the relationship between the predicted OS and the observed OS probability. Comparison of the nomogram and traditional AJCC TNM staging system was carried out through the R 'Hmisc' package and tested by $\mathrm{C}$-index. The C-index (range: $0.5-1$ ) reflected the discrimination capacity of the model and larger values indicate better discrimination among different survival outcome. Additionally, decision curve analysis was employed to assess the clinical net benefit of the predictive model. After calculating the total nomogram scores for patients in training and validation cohort, then determining the optimal cutoff values by $\mathrm{X}$-tile, individuals were separated into low-risk or high-risk subgroups. Survival estimation was accomplished by the Kaplan-Meier (KM) method and differences between survival curves were analysed by log-rank test. All analyses were performed by SPSS26.0 for windows (IBM Corporation; Chicago, IL, USA) and R version3.6.2 (Institute for Statistics and Mathematics; Vienna, Austria) using 'rms', 'survival', 'Hmisc', and 'stdca' packages. Two-sided P value less than .05 was considered statistically significant.

\section{Results}

\section{The characteristics of Patients with pCCA}

A sum of 940 eligible patients were included in the whole study cohort, which was then selected at random into the training set and validation set. Detailed process for patient selection was shown in Figure.1. The demographic and clinicopathological characteristics of all patients were presented in Table 1. All variables were similar in both sets. In the whole cohort, there were 876 events (death) over the 
median follow-up of 79 months (interquartile range: 52-123 months). The median OS was 4 months (95\%Cl: 3.3-4.7 months), with 1-, 2-, and 3-year OS rates of $26.2 \%, 14.4 \%$ and $8.4 \%$, respectively. 
Table 1

Demographic and Clinicopathological Characteristics of Patients with perihilar cholangiocarcinoma.

\begin{tabular}{|c|c|c|c|c|}
\hline \multirow[t]{2}{*}{ Variables } & All patients & Training set & Validation set & \multirow[t]{2}{*}{ P-value } \\
\hline & $\mathrm{n}=940(\%)$ & $n=658(\%)$ & $n=282(\%)$ & \\
\hline Age & & & & 0.646 \\
\hline$\leq 68$ & $373(39.7)$ & $263(40.0)$ & $110(39.0)$ & \\
\hline $69-84$ & $381(40.5)$ & $270(41.0)$ & $111(39.4)$ & \\
\hline$\geq 85$ & 186(19.8) & $125(19.0)$ & $61(21.6)$ & \\
\hline Sex & & & & 0.742 \\
\hline Male & $519(55.2)$ & $361(54.9)$ & $158(56.0)$ & \\
\hline Female & $421(44.8)$ & $297(45.1)$ & $124(44.0)$ & \\
\hline Race & & & & 0.420 \\
\hline White & 744(79.1) & $520(79.0)$ & $224(79.4)$ & \\
\hline Black & $64(6.8)$ & $49(7.4)$ & $15(5.3)$ & \\
\hline Other & $132(14.0)$ & $89(13.5)$ & $43(15.2)$ & \\
\hline Marital status & & & & 0.459 \\
\hline Married & 496(52.8) & $343(52.1)$ & 153(54.3) & \\
\hline Unmarried & $406(43.2)$ & $291(44.2)$ & 115(40.8) & \\
\hline Unknown & $38(4.0)$ & $24(3.6)$ & $14(5.0)$ & \\
\hline Grade & & & & 0.804 \\
\hline$\nabla+\square$ & $99(10.5)$ & 72(10.9) & $27(9.6)$ & \\
\hline$\nabla+\square$ & $64(6.8)$ & $44(6.7)$ & $20(7.1)$ & \\
\hline Unknown & 777(82.7) & $542(82.4)$ & 235(83.3) & \\
\hline SEER Stage & & & & 0.186 \\
\hline Localized & $238(25.3)$ & $160(24.3)$ & 78(27.7) & \\
\hline Regional & $312(33.2)$ & 222(33.7) & $90(31.9)$ & \\
\hline Distant & $173(18.4)$ & 131(19.9) & $42(14.9)$ & \\
\hline Unknown & $217(23.1)$ & $145(22.0)$ & $72(25.5)$ & \\
\hline T stage & & & & 0.338 \\
\hline
\end{tabular}




\begin{tabular}{|c|c|c|c|c|}
\hline \multirow[t]{2}{*}{ Variables } & All patients & Training set & Validation set & P-value \\
\hline & $n=940(\%)$ & $\mathrm{n}=658(\%)$ & $n=282(\%)$ & \\
\hline T1 & $284(30.2)$ & 188(28.6) & $96(34.0)$ & \\
\hline $\mathrm{T} 2$ & $73(7.8)$ & $51(7.8)$ & $22(7.8)$ & \\
\hline T3 & 191(20.3) & 139(21.1) & $52(18.4)$ & \\
\hline $\mathrm{T} 4$ & 101(10.7) & 77(11.7) & $24(8.5)$ & \\
\hline TX & 291(31.0) & 203(30.9) & $88(31.2)$ & \\
\hline N stage & & & & 0.338 \\
\hline NO & $586(62.3)$ & $415(63.1)$ & $171(60.6)$ & \\
\hline N1 & 158(16.8) & 114(17.3) & $44(15.6)$ & \\
\hline NX & 196(20.9) & $129(19.6)$ & $67(23.8)$ & \\
\hline M stage & & & & 0.153 \\
\hline MO & 635(67.6) & $444(67.5)$ & $191(67.7)$ & \\
\hline M1 & 160(17.0) & $120(18.2)$ & $40(14.2)$ & \\
\hline $\mathrm{MX}$ & 145(15.4) & $94(14.3)$ & $51(18.1)$ & \\
\hline Surgery & & & & 0.182 \\
\hline Yes & $113(12.0)$ & 73(11.1) & $40(14.2)$ & \\
\hline No & $827(88.0)$ & $585(88.9)$ & $242(85.8)$ & \\
\hline Radiation & & & & 0.279 \\
\hline Yes & 166(17.7) & 122(18.5) & $44(15.6)$ & \\
\hline No & 774(82.3) & $536(81.5)$ & $238(84.4)$ & \\
\hline Chemotherapy & & & & 0.836 \\
\hline Yes & $259(27.6)$ & $180(27.4)$ & $79(28.0)$ & \\
\hline No & $681(72.4)$ & $478(72.6)$ & $203(72.0)$ & \\
\hline
\end{tabular}

\section{Independent predictors for OS in the training set}

As described in Table 2, through the univariate analysis, a total of 10 covariates were related to OS $(\mathrm{P}<$ $0.05)$, while gender and race had no correlation with survival. Statistically significant variables in the univariate Cox analysis were combined into the subsequent analysis. Following adjustment for other covariates, multivariable analysis indicated that age, summary stage, surgery, chemotherapy as well as radiation remained independently connected with OS. 
Table 2

Univariate and multivariable analysis for OS in the training cohort.

\begin{tabular}{|c|c|c|c|c|}
\hline \multirow[t]{2}{*}{ covariates } & \multicolumn{2}{|l|}{ Univariate analysis } & \multicolumn{2}{|l|}{ Multivariate analysis } \\
\hline & $\mathrm{HR}(95 \% \mathrm{Cl})$ & P-value & $\mathrm{HR}(95 \% \mathrm{Cl})$ & P-value \\
\hline \multicolumn{5}{|l|}{ Age } \\
\hline$\leq 68$ & 1 & & 1 & \\
\hline $69-84$ & $1.457(1.220-1.741)$ & $<0.001$ & $1.231(1.017-1.489)$ & $0.033^{\star}$ \\
\hline$\geq 85$ & $2.301(1.840-2.877)$ & $<0.001$ & $1.722(1.347-2.201)$ & $<0.001 *$ \\
\hline Sex & & & $\mathrm{NI}$ & \\
\hline Male & 1 & & & \\
\hline Female & $1.152(0.982-1.351)$ & 0.081 & & \\
\hline Race & & & $\mathrm{NI}$ & \\
\hline White & 1 & & & \\
\hline Black & $1.251(0.924-1.694)$ & 0.147 & & \\
\hline Other & $0.814(0.641-1.034)$ & 0.092 & & \\
\hline \multicolumn{5}{|c|}{ Marital status } \\
\hline Married & 1 & & & \\
\hline Unmarried & $1.411(1.199-1.660)$ & $<0.001$ & & 0.316 \\
\hline \multicolumn{5}{|l|}{ Grade } \\
\hline$\nabla+\nabla$ & 1 & & & \\
\hline$\nabla+\square$ & $1.254(0.840-1.872)$ & 0.268 & & 0.687 \\
\hline \multicolumn{5}{|c|}{ SEER Stage } \\
\hline Localized & 1 & & 1 & \\
\hline Regional & $0.825(0.667-1.021)$ & 0.077 & $1.355(1.078-1.704)$ & $0.009 *$ \\
\hline Distant & $1.378(1.088-1.744)$ & 0.008 & $1.726(1.345-2.214)$ & $<0.001 *$ \\
\hline Unknown & $1.375(1.092-1.731)$ & 0.007 & $1.177(0.933-1.484)$ & 0.170 \\
\hline \multicolumn{5}{|l|}{ T stage } \\
\hline T1 & 1 & & & \\
\hline
\end{tabular}

$H R$, hazard ratio; $C l$, confidence interval; $N I$, not included. *significance values. 


\begin{tabular}{|c|c|c|c|c|}
\hline covariates & Univariate analysis & & Multivariate analysis & \\
\hline $\mathrm{T} 2$ & $0.496(0.353-0.695)$ & $<0.001$ & & 0.088 \\
\hline T3 & $0.788(0.627-0.990)$ & 0.041 & & 0.328 \\
\hline T4 & $0.851(0.647-1.119)$ & 0.248 & & 0.347 \\
\hline \multicolumn{5}{|l|}{ N stage } \\
\hline NO & 1 & & & \\
\hline $\mathrm{N} 1$ & $0.941(0.760-1.166)$ & 0.580 & & 0.754 \\
\hline \multicolumn{5}{|l|}{ M stage } \\
\hline MO & 1 & & & \\
\hline M1 & $1.543(1.257-1.896)$ & $<0.001$ & & 0.965 \\
\hline \multicolumn{5}{|l|}{ Surgery } \\
\hline No & 1 & & 1 & \\
\hline Yes & $0.347(0.260-0.464)$ & $<0.001$ & $0.403(0.296-0.548)$ & $<0.001 *$ \\
\hline \multicolumn{5}{|l|}{ Radiation } \\
\hline No & 1 & & 1 & \\
\hline Yes & $0.508(0.412-0.626)$ & $<0.001$ & $0.759(0.599-0.962)$ & $0.022^{\star}$ \\
\hline \multicolumn{5}{|c|}{ Chemotherapy } \\
\hline No & 1 & & 1 & \\
\hline Yes & $0.499(0.416-0.598)$ & $<0.001$ & $0.612(0.493-0.760)$ & $<0.001 *$ \\
\hline
\end{tabular}

The predictive nomogram model incorporating all significant prognostic factors identified by Cox regression analysis was built as presented in Figure.2. The length of each respective point scale indicates the extent of contribution to survival outcome. As demonstrated in the nomogram, surgery treatment shared the most contribution, followed by the summary stage and age. In addition, presence or absence of radiation also had a moderate effect on the prognosis. In terms of the internal validation, the nomogram had a C-index of 0.703 ( $95 \% \mathrm{Cl}$ : $0.680-0.725)$, as could be seen in Figure.3, the calibration plots for 1-, 2-, and 3-year OS probabilities indicated high consistency between the nomogram-predicted and the actual observations. Regarding the external validation, the nomogram still exhibited good accuracy, supported by the C-index of 0.718 (95\% Cl: $0.684-0.752)$. Calibration curves for external validation presented in Figure. 3 still confirmed satisfactory agreements between OS predictions and the 
actual observations. In a word, excellent discrimination and calibration in internal and external validations guaranteed the reliability and repeatability of the established nomogram.

\section{Comparison between the nomogram and AJCC TNM system}

Comparison of the discriminative ability of nomogram and traditional TNM staging system was executed by calculating corresponding $\mathrm{C}$-indies. In the training cohort, our model has a notably higher value than that of TNM $(0.703$ vs $0.572, P<0.001)$, which was then verified in the validation set $(0.718$ vs $0.588, P<$ 0.001). To further validate and compare the superiority of the nomogram and TNM system in clinical utility, decision curve analysis was introduced to our study. As depicted in Figure.4, the nomogram yielded remarkably more benefits in foreseeing 1-year, 2-year and 3-year OS than TNM staging system did over a relatively broad range of threshold probabilities.

\section{The performance of nomogram on risk group stratification}

Relying on the nomogram total points calculated for each patient and the optimal cut-off values detected by the X-tile analysis, patients were categorized into the low- and high-risk subgroups. In this study, the cases were divided as the low- (less than 214 total points) and the high-risk group (greater than or equal to 214 total points) respectively. The KM curves were plotted in Figure.5, which illustrated that patients in lower risk had significantly higher OS probabilities in comparison with the ones in high-risk group. The above results denoted that the nomogram was reliable in predicting the probability of OS for Klatskin tumors patients and aiding in gaining better equivalence between study arms for clinical study design.

\section{Discussion}

Most pCCA are diagnosed at an advanced stage [26]. Therefore, individualized survival evaluation via easily accessible measures for patients is of importance. In the current study, five independent determinants were screened through univariate and multivariate Cox proportional hazard regression analysis, namely as age, surgery, chemotherapy, radiation and summary stage. We developed the nomogram with these five factors to predict 1-, 2-, and 3-year OS probabilities of pCCA, which allows physicians to predict their patients' prognosis in an easy-to-implement way. It's in agreements between the nomogram-predicted OS and the observed OS as evidenced by C-indies of 0.703 and 0.718 in both training and validation sets respectively. We had an additional interpretation that the calibration for 3-year OS was slightly reduced when externally validated, whereas the discrimination was outstanding. Tumor characteristics, management strategies and the differences within patients may be responsible for the suboptimal calibration in validation set. In addition, survival curves of distinct risk groups manifested the significantly statistical difference, indicating the feasibility of the constructed monograph.

As our results indicated, age was significantly correlated with OS rates, with older age corresponding to shorter OS, which was in accordance with the published studies $[27,28]$. To obtain the most significant difference between age groups, optimal cut-off values derived from X-tile analysis were applied to both 
the training and validation cohort. It's recognized that patients with older age have worse surgery or other treatment tolerance and other comorbidities that overall survival might be negatively affected. However, Koerkamp et al [29] revealed that age was not a significant factor to predict disease-specific survival (DSS). We speculated that deaths from some other competing risks like cardiovascular events may partially explain the result. Interestingly, in the nomogram presented by Chen et al [30], age was also related to the OS, whereas age at 55 years old indicated the lowest OS rate, with the OS rate conversely increasing within the age between 55 to 85 . The underlying reason for the contradiction remained unclear.

SEER summary stage is a basic method of determining how far a cancer has extended from its point of origin, mainly comprising local, regional, and distant stage. What's more, the regional stage consists of three detailed classifications, including direct extension only, lymph nodes involved only, both direct extension and lymph node involvement. The present study detected the prognostic value of summary stage in association with OS probabilities, which was similar to the results published by Qi et al [28]. Compared to the cases at regional and distant stage, only tumors at the localized stage had better prognosis.

With regards to the treatments, other two treatment modalities of chemotherapy and radiation also demonstrated significant correlation with survival outcome besides surgery. Surgery with curative intent can prolong the median OS of patients from 8 months to 40 months [31]. Nevertheless, only a confined portion of patients met the eligibility for surgical treatment, with the addition of high recurrence rate after the resection, so options like chemotherapy or radiation should be considered in these cases for adjuvant or palliative treatment. Controversy also exists in the correlation between chemotherapy or radiation and OS. Chen et al [30] proposed that chemotherapy and radiotherapy was not related to the OS, while they were both independent prognostic factors in our study, thus integrated in the nomogram. In reference of a randomized trial [32], adjuvant capecitabine chemotherapy should be given to patients with the resected cholangiocarcinoma for 6 months duration [33], with a dose of $1,250 \mathrm{mg} / \mathrm{m}^{2}$ twice a day in every 3-week cycle. In addition, several evidences disclosed that there were potential benefits of chemotherapy treatment in both preoperative and postoperative Klatskin tumors [34-36]. The regimen of gemcitabine and cisplatin was recommended for inoperable or postoperative pCCA patients [4,37], but detailed chemotherapy regimen information were unavailable in SEER database. Patients with extrahepatic cholangiocarcinoma may be offered chemoradiation therapy, which was administered with a dose of $45 \mathrm{~Gy}$ to regional lymphatics and 54 to $59.4 \mathrm{~Gy}$ to the tumor bed [38, 39]. With respect to the radiotherapy for perihilar cholangiocarcinoma, Leng et al [40] argued that adjuvant radiotherapy was of no association with survival improvement for resected pCCA groups. A retrospective study [41] of SEER dataset also concluded that radiation modality had no therapeutic benefits for pCCA. But in the light of some reviews $[42,43]$, external beam and endoluminal radiation therapy was an option for unresectable hilar cholangiocarcinoma. In general, although the efficacy of radiotherapy for pCCA was still in dispute, it was fit into our final model on multivariate analysis. Methods of radiation mainly comprised the beam radiation, radioactive implants (including brachytherapy), and combination of beam with implants or 
isotopes. Additionally, appropriate dosing, timing and radioactive source were needed for further investigation.

There have been several prognostic models for pCCA reported, including a point scoring system and three nomograms [28-30,44]. As specified in several previous studies, lymph node counts and lymph node metastasis were often identified as independent predictors for PCCA $[45,46]$. For instance, lymph node ratio (LNR) was often utilized to develop the nomogram for biliary tract system diseases $[47,48]$. While in our study, LNR is hard to formulate due to the missing data, and AJCC N stage failed to enter into the final model with statistical insignificance. Compared to the scoring system, the current study confirmed the prognostic roles of age and chemoradiation in patients with PCCA, and the proposed nomogram could work for a scoring system and a visualized prediction approach at the same time. Concerning the nomograms developed for patients with resected pCCA by Koerkamp et al [29] and Chen et al [30], our model did not include portal vein or hepatic artery involvement, tumor pathological differentiation and lymph node status as well as margin status, which might influence the prognosis of the pCCA patients, but SEER summary stage could equivalently reflect these tumor characteristics to a great extent. Moreover, we have encompassed the chemotherapy and radiation factors rarely mentioned in other studies. With regard to a recently published nomogram with C-index of 0.651 , it was only based on 317 patients from the SEER database [28], without consideration of chemotherapy and radiation information. In contrast, our model was based on a large cohort of cases, and has a comparable discrimination and calibration to those relevant models.

Despite the good performance and easy-to-use of our constructed monograph as a prognostic model, some limitations in our study should be noted. For instance, due to the retrospective nature, the nomogram establishment was based on the data from the SEER program that selection bias is a potential concern. Furthermore, some important clinicopathological parameters and widely used tumor biomarkers for pCCA, such as surgical margin status, the information of serum carbohydrate antigen 19 - 9 (CA199), carcinoembryonic antigen (CEA) and vascular encasement were unavailable in SEER database. In addition, many promising options like interventional therapy [49], immunotherapy [50] and targeted treatment [51] are enjoying increasing popularities among pCCA patients, but it is a pity that they have been inaccessible in SEER program until now. As a user-friendly method for prognosis prediction and decision-making, there is difficulty in providing everlastingly excellent prognosis prediction in clinical practice, because not all significant prognostic factors were embodied in this tool.

\section{Conclusions}

In conclusion, the proposed nomogram in our study could predict the OS of patients with Klatskin tumors effectively and efficiently, which could be utilized for individualized prognosis prediction and patient stratification. Further large-scale and prospective randomized studies are warranted to determine the clinical applicability of the model.

\section{Abbreviations}


CCA: Cholangiocarcinoma; pCCA:Perihilar cholangiocarcinoma; OS:Overall Survival; SEER:The Surveillance, Epidemiology, and End Results; AJCC:The American Joint Committee on Cancer; TNM:Tumor-Node-Metastasis; Cl:Confidence interval; HR:Hazard ratio; C-index:Harrell's concordance index

\section{Declarations}

\section{Ethics approval and consent to participate:}

All procedures performed in studies involving human participants were in accordance with the ethical standards of the institutional and/or national research committee and with the 1964 Helsinki Declaration and its later amendments or comparable ethical standards.

\section{Consent for publication:}

Not applicable

\section{Availability of data and materials:}

The datasets generated and analysed during the current study are available in the SEER repository, https://seer.cancer.gov/.

\section{Competing interests:}

The authors declare that they have no competing interests

\section{Funding:}

This study was supported by a grant from Nantong "226 Project" and Excellent Key Teachers in the "Qing Lan Project" of Jiangsu Colleges and Universities.

\section{Authors' contributions:}

Chendong Wang and Huiqun Wu designed the study. Chendong Wang,Huanyu Gong and Zhiyuan Zhang accessed databases and gathered all variables. Chendong Wang, Huanyu Gong, and Danzhou Fang assumed responsibility for completeness and accuracy of data analysis and interpretation. The manuscript was written by Chendong Wang. All authors have read and approved the final manuscript.

\section{Acknowledgements:}


The authors would like to thank the Surveillance, Epidemiology, and End Results (SEER) Program for providing open access for research.

\section{References}

1. Blechacz B. Cholangiocarcinoma. Current Knowledge and New Developments. Gut Liver. 2017;11(1):13-26.

2. Kendall T, Verheij J, Gaudio E, et al. Anatomical, histomorphological and molecular classification of cholangiocarcinoma. Liver Int. 2019;39(Suppl 1):7-18.

3. Nakeeb A, Pitt HA, Sohn TA, et al. Cholangiocarcinoma. A spectrum of intrahepatic, perihilar, and distal tumors. Ann Surg. 1996;224(4):463-73. discussion $73-5$.

4. Razumilava N, Gores GJ. Cholangiocarcinoma. Lancet. 2014;383(9935):2168-79.

5. Cassani LS, Chouhan J, Chan C, et al. Biliary Decompression in Perihilar Cholangiocarcinoma Improves Survival: A Single-Center Retrospective Analysis. Digestive diseases sciences. 2019;64(2):561-9.

6. Cillo U, Fondevila C, Donadon M, et al. Surgery for cholangiocarcinoma. Liver Int. 2019;39 Suppl $1(143-55$.

7. Groot Koerkamp B, Wiggers JK, Allen PJ, et al. Recurrence Rate and Pattern of Perihilar Cholangiocarcinoma after Curative Intent Resection. J Am Coll Surg. 2015;221(6):1041-9.

8. Nassour I, Mokdad AA, Porembka MR, et al. Adjuvant Therapy Is Associated With Improved Survival in Resected Perihilar Cholangiocarcinoma: A Propensity Matched Study. Ann Surg Oncol. 2018;25(5):1193-201.

9. Palmieri LJ, Lavole J, Dermine S, et al. The choice for the optimal therapy in advanced biliary tract cancers: Chemotherapy, targeted therapies or immunotherapy. Pharmacol Ther. 2020:107517.

10. Zhang W, Yang ZQ, Shi HB, et al. Placement of ${ }^{125}$ I seed strands and stents for a type IV Klatskin tumor. World journal of gastroenterology. 2015;21(1):373-6.

11. Bismuth $\mathrm{H}$, Castaing $\mathrm{D}$, Traynor $\mathrm{O}$. Resection or palliation: priority of surgery in the treatment of hilar cancer. World J Surg. 1988;12(1):39-47.

12. Bismuth $\mathrm{H}$, Corlette MB. Intrahepatic cholangioenteric anastomosis in carcinoma of the hilus of the liver. Surg Gynecol Obstet. 1975;140(2):170-8.

13. Jarnagin WR, Fong Y, DeMatteo RP, et al. Staging, resectability, and outcome in 225 patients with hilar cholangiocarcinoma. Ann Surg. 2001;234(4):507-17. discussion 17 - 9.

14. Zaydfudim VM, Clark CJ, Kendrick ML, et al. Correlation of staging systems to survival in patients with resected hilar cholangiocarcinoma. Am J Surg. 2013;206(2):159-65.

15. Suarez-Munoz MA, Fernandez-Aguilar JL, Sanchez-Perez B, et al. Risk factors and classifications of hilar cholangiocarcinoma. World J Gastrointest Oncol. 2013;5(7):132-8.

16. Chaiteerakij R, Harmsen WS, Marrero $C R$, et al. A new clinically based staging system for perihilar cholangiocarcinoma. Am J Gastroenterol. 2014;109(12):1881-90. 
17. Jalali A, Alvarez-Iglesias A, Roshan $D$, et al. Visualising statistical models using dynamic nomograms. PloS one. 2019;14(11):e0225253.

18. Wang Y, Li J, Xia Y, et al. Prognostic nomogram for intrahepatic cholangiocarcinoma after partial hepatectomy. J Clin Oncol. 2013;31(9):1188-95.

19. Dong D, Tang L, Li ZY, et al. Development and validation of an individualized nomogram to identify occult peritoneal metastasis in patients with advanced gastric cancer. Ann Oncol. 2019;30(3):431-8.

20. Pan X, Yang W, Chen $Y$, et al. Nomogram for predicting the overall survival of patients with inflammatory breast cancer: A SEER-based study. Breast. 2019;47:56-61.

21. Liang W, Zhang L, Jiang G, et al. Development and validation of a nomogram for predicting survival in patients with resected non-small-cell lung cancer. J Clin Oncol. 2015;33(8):861-9.

22. Diao JD, Ma LX, Sun MY, et al. Construction and validation of a nomogram to predict overall survival in patients with inflammatory breast cancer. Cancer medicine. 2019;8(12):5600-8.

23. Hanrahan EO, Gonzalez-Angulo AM, Giordano SH, et al. Overall survival and cause-specific mortality of patients with stage T1a,bNOMO breast carcinoma. J Clin Oncol. 2007;25(31):4952-60.

24. Harrell FE Jr, Lee KL, Mark DB. Multivariable prognostic models: issues in developing models, evaluating assumptions and adequacy, and measuring and reducing errors. Stat Med. 1996;15(4):361-87.

25. Pencina MJ, D'Agostino RB. Overall $C$ as a measure of discrimination in survival analysis: model specific population value and confidence interval estimation. Stat Med. 2004;23(13):2109-23.

26. Banales JM, Cardinale V, Carpino G, et al. Expert consensus document: Cholangiocarcinoma: current knowledge and future perspectives consensus statement from the European Network for the Study of Cholangiocarcinoma (ENS-CCA). Nat Rev Gastroenterol Hepatol. 2016;13(5):261-80.

27. Zhang X, Liu H. Klatskin Tumor: A Population-Based Study of Incidence and Survival. Med Sci Monit. 2019;25:4503-12.

28. Qi F, Zhou B, Xia J. Nomograms predict survival outcome of Klatskin tumors patients. PeerJ. 2020;8:e8570.

29. Groot Koerkamp B, Wiggers JK, Gonen M, et al. Survival after resection of perihilar cholangiocarcinoma-development and external validation of a prognostic nomogram. Ann Oncol. 2015;26(9):1930-5.

30. Chen P, Li B, Zhu Y, et al. Establishment and validation of a prognostic nomogram for patients with resectable perihilar cholangiocarcinoma. Oncotarget. 2016;7(24):37319-30.

31. Groot Koerkamp B, Jarnagin WR. Surgery for perihilar cholangiocarcinoma. Br J Surg. 2018;105(7):771-2.

32. Primrose JN, Fox RP, Palmer DH, et al. Capecitabine compared with observation in resected biliary tract cancer (BILCAP): a randomised, controlled, multicentre, phase 3 study. Lancet Oncol. 2019;20(5):663-73. 
33. Shroff RT, Kennedy EB, Bachini M, et al. Adjuvant Therapy for Resected Biliary Tract Cancer: ASCO Clinical Practice Guideline. J Clin Oncol. 2019;37(12):1015-27.

34. Baltatzis M, Jegatheeswaran S, Siriwardena AK. Neoadjuvant chemoradiotherapy before resection of perihilar cholangiocarcinoma: A systematic review. Hepatobiliary Pancreat Dis Int. 2020.

35. Murakami Y, Uemura K, Sudo T, et al. Gemcitabine-based adjuvant chemotherapy improves survival after aggressive surgery for hilar cholangiocarcinoma. Journal of gastrointestinal surgery: official journal of the Society for Surgery of the Alimentary Tract. 2009;13(8):1470-9.

36. Ramirez-Merino N, Aix SP, Cortes-Funes H. Chemotherapy for cholangiocarcinoma: An update. World J Gastrointest Oncol. 2013;5(7):171-6.

37. Valle J, Wasan $\mathrm{H}$, Palmer $\mathrm{DH}$, et al. Cisplatin plus gemcitabine versus gemcitabine for biliary tract cancer. N Engl J Med. 2010;362(14):1273-81.

38. Ben-Josef E, Guthrie KA, El-Khoueiry AB, et al. SWOG S0809: A Phase II Intergroup Trial of Adjuvant Capecitabine and Gemcitabine Followed by Radiotherapy and Concurrent Capecitabine in Extrahepatic Cholangiocarcinoma and Gallbladder Carcinoma. J Clin Oncol. 2015;33(24):2617-22.

39. Elganainy D, Holliday EB, Taniguchi CM, et al. Dose escalation of radiotherapy in unresectable extrahepatic cholangiocarcinoma. Cancer medicine. 2018;7(10):4880-92.

40. Leng KM, Liu YP, Wang ZD, et al. Results of adjuvant radiation therapy for locoregional perihilar cholangiocarcinoma after curative intent resection. OncoTargets therapy. 2017;10:2257-66.

41. Sharma P, Yadav S. Demographics, tumor characteristics, treatment, and survival of patients with Klatskin tumors. Annals of gastroenterology. 2018;31(2):231-6.

42. Mansour JC, Aloia TA, Crane $\mathrm{CH}$, et al. Hilar cholangiocarcinoma: expert consensus statement. HPB: the official journal of the International Hepato Pancreato Biliary Association. 2015;17(8):691-9.

43. Rizvi S, Khan SA, Hallemeier CL, et al. Cholangiocarcinoma - evolving concepts and therapeutic strategies. Nat Rev Clin Oncol. 2018;15(2):95-111.

44. Kaiser GM, Paul A, Sgourakis G, et al. Novel prognostic scoring system after surgery for Klatskin tumor. Am Surg. 2013;79(1):90-5.

45. Gao Y, Xu D, Wu YS, et al. Increasing negative lymph node count is independently associated with improved long-term survival in resectable perihilar cholangiocarcinomas. Medicine. 2019;98(15):e14943.

46. Hakeem AR, Marangoni G, Chapman SJ, et al. Does the extent of lymphadenectomy, number of lymph nodes, positive lymph node ratio and neutrophil-lymphocyte ratio impact surgical outcome of perihilar cholangiocarcinoma? Eur J Gastroenterol Hepatol. 2014;26(9):1047-54.

47. Ma K, Dong B, Wang L, et al. Nomograms for predicting overall survival and cancer-specific survival in patients with surgically resected intrahepatic cholangiocarcinoma. Cancer management research. 2019;11:6907-29.

48. He C, Cai Z, Zhang Y, et al. Prognostic Model to Predict Cancer-Specific Survival for Patients With Gallbladder Carcinoma After Surgery: A Population-Based Analysis. Front Oncol. 2019;9:1329. 
49. Wang X, Hu J, Cao G, et al. Phase II Study of Hepatic Arterial Infusion Chemotherapy with Oxaliplatin and 5-Fluorouracil for Advanced Perihilar Cholangiocarcinoma. Radiology. 2017;283(2):580-9.

50. Rizvi S, Gores GJ. Emerging molecular therapeutic targets for cholangiocarcinoma. Journal of hepatology. 2017;67(3):632-44.

51. Valle JW. Targeted therapy for cholangiocarcinoma. Lancet Gastroenterol Hepatol. 2019;4(9):661-2.

\section{Figures}

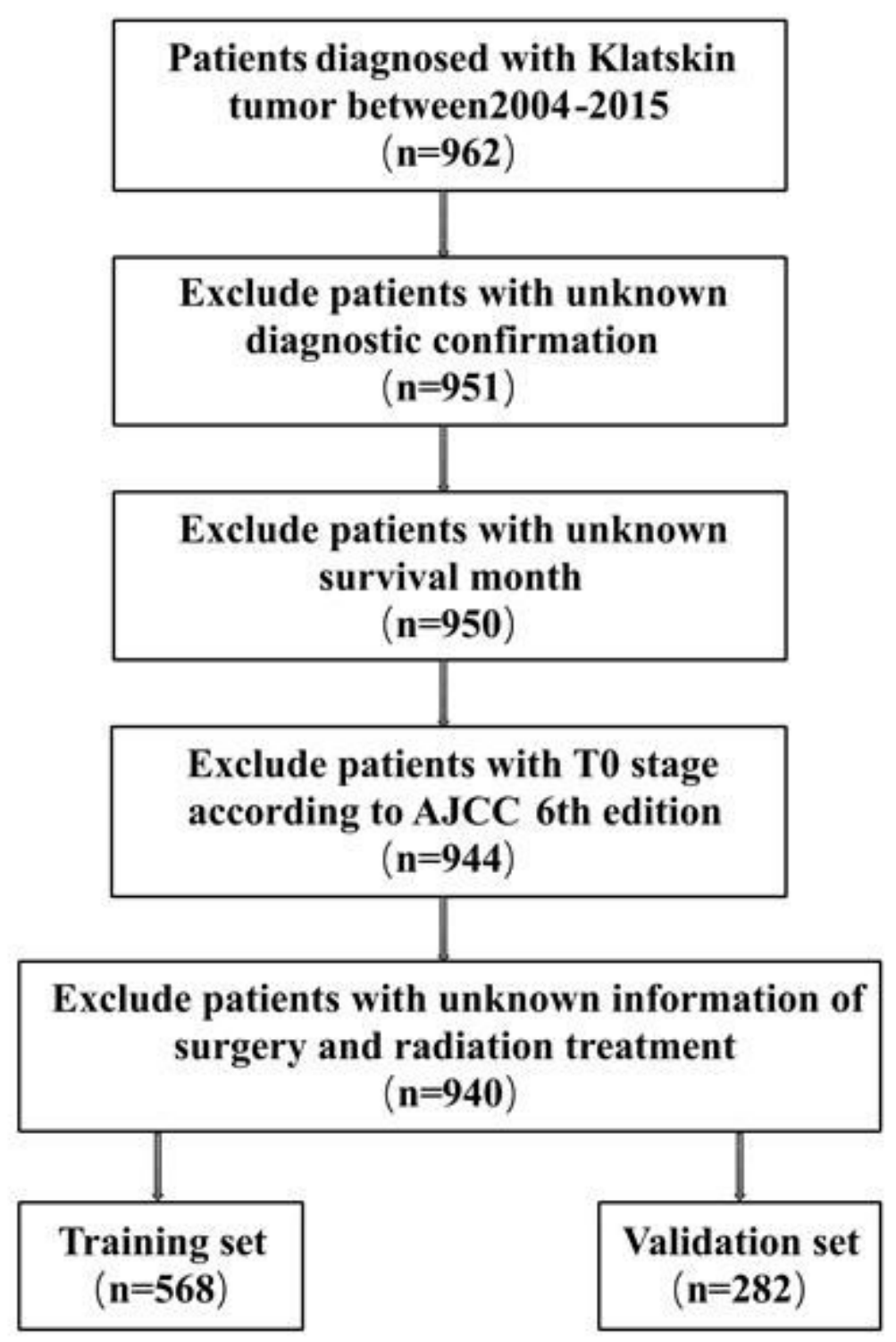

Figure 1

The flowchart of patient selection. 


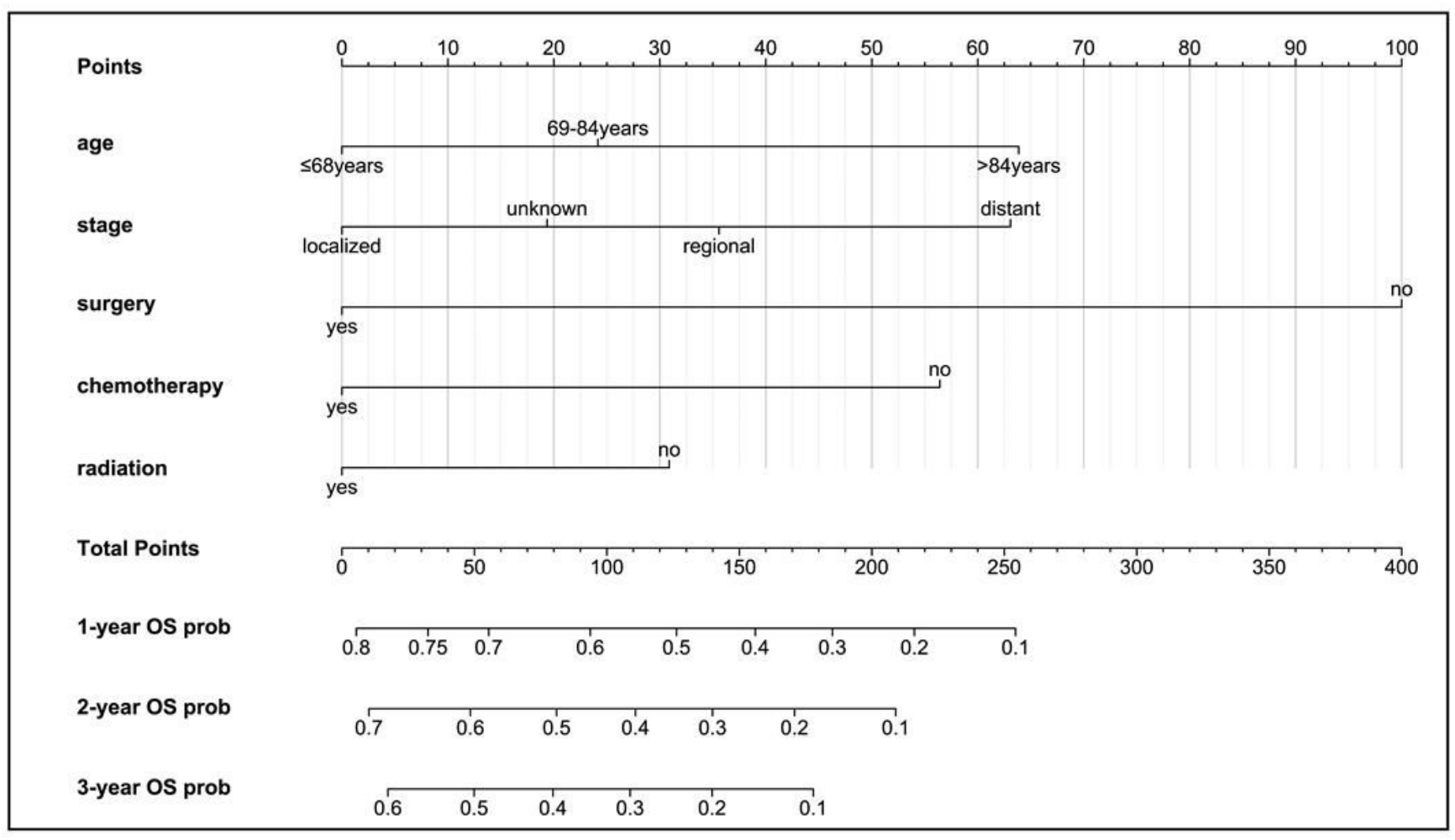

\section{Figure 2}

The constructed nomogram for predicting 1-year, 2-year, and 3-year OS of patients with perihilar cholangiocarcinoma (CCA). OS prob: overall survival probability; stage: SEER summary stage. Each subtype of these variants has a corresponding score on the points scale. By adding up the total score and locating it on the total points scale, then drawing a vertical line downward to the survival axes, the estimated probability of 1-, 2-, and 3-year OS can be easily determined. 
(a)

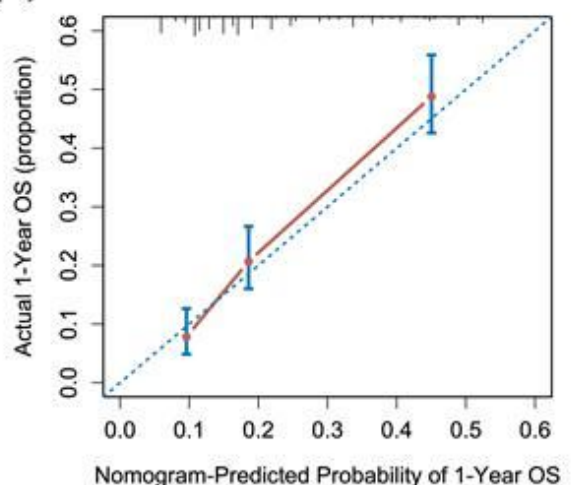

(d)

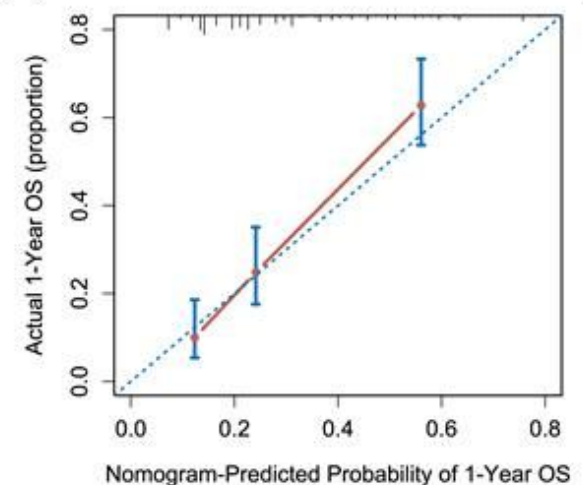

(b)

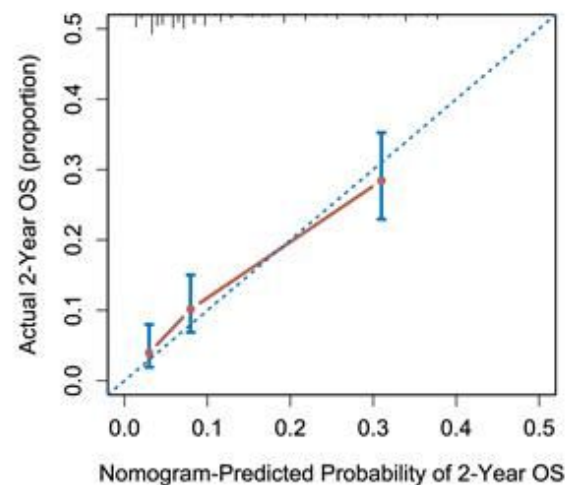

(e)

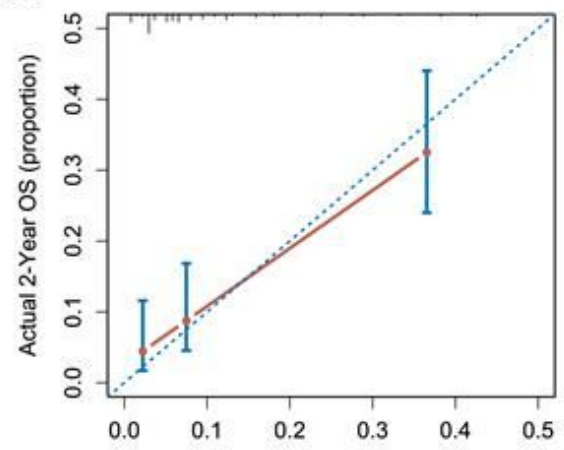

Nomogram-Predicted Probability of 2-Year OS (c)

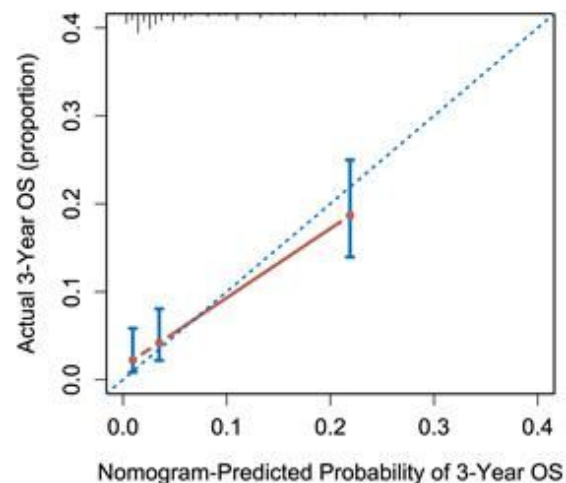

(f)

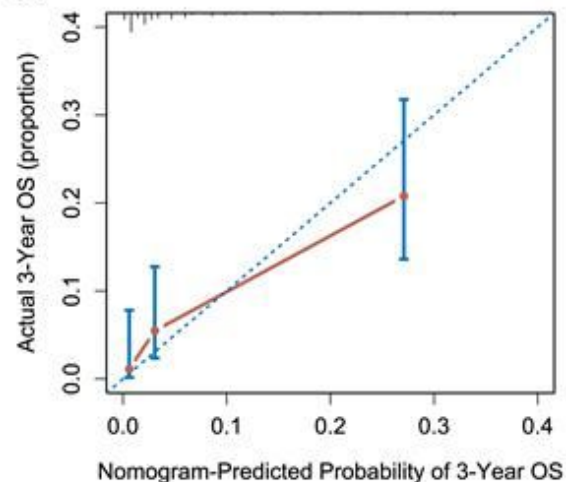

Figure 3

The calibration curve for predicting patient's OS at (a) 1-year, (b) 2-year and (c) 3-year in the training cohort and at (d) 1-year, (e) 2-year and (f) 3-year in the validation cohort. Note: Nomogram-predicted probability of OS was plotted on the x-axis; actual OS was plotted on the y-axis. A plot along the 45degree line indicated a perfect calibration model in which the predicted probabilities were identical to the actual outcomes.

(a)

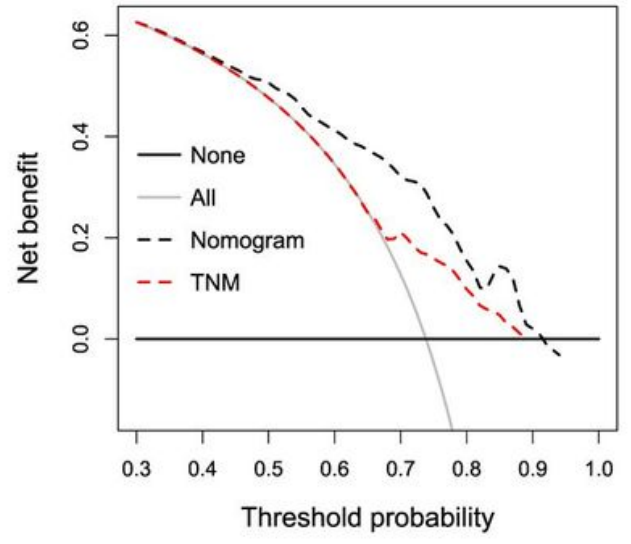

(b)

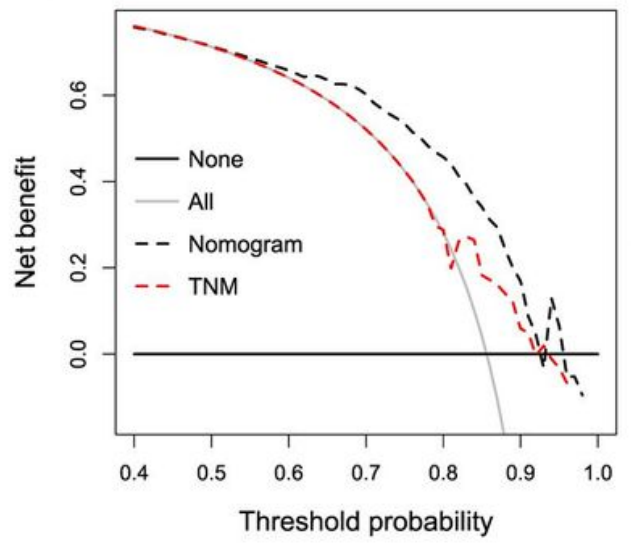

(c)

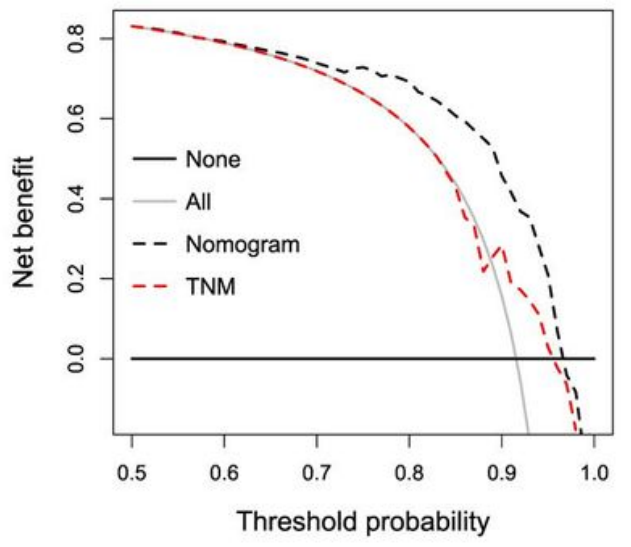


Figure 4

The decision curve of the constructed nomogram and TNM for predicting (a) 1-year OS; (b) 2-year OS; (c) 3-year OS of patients with perihilar holangiocarcinoma.

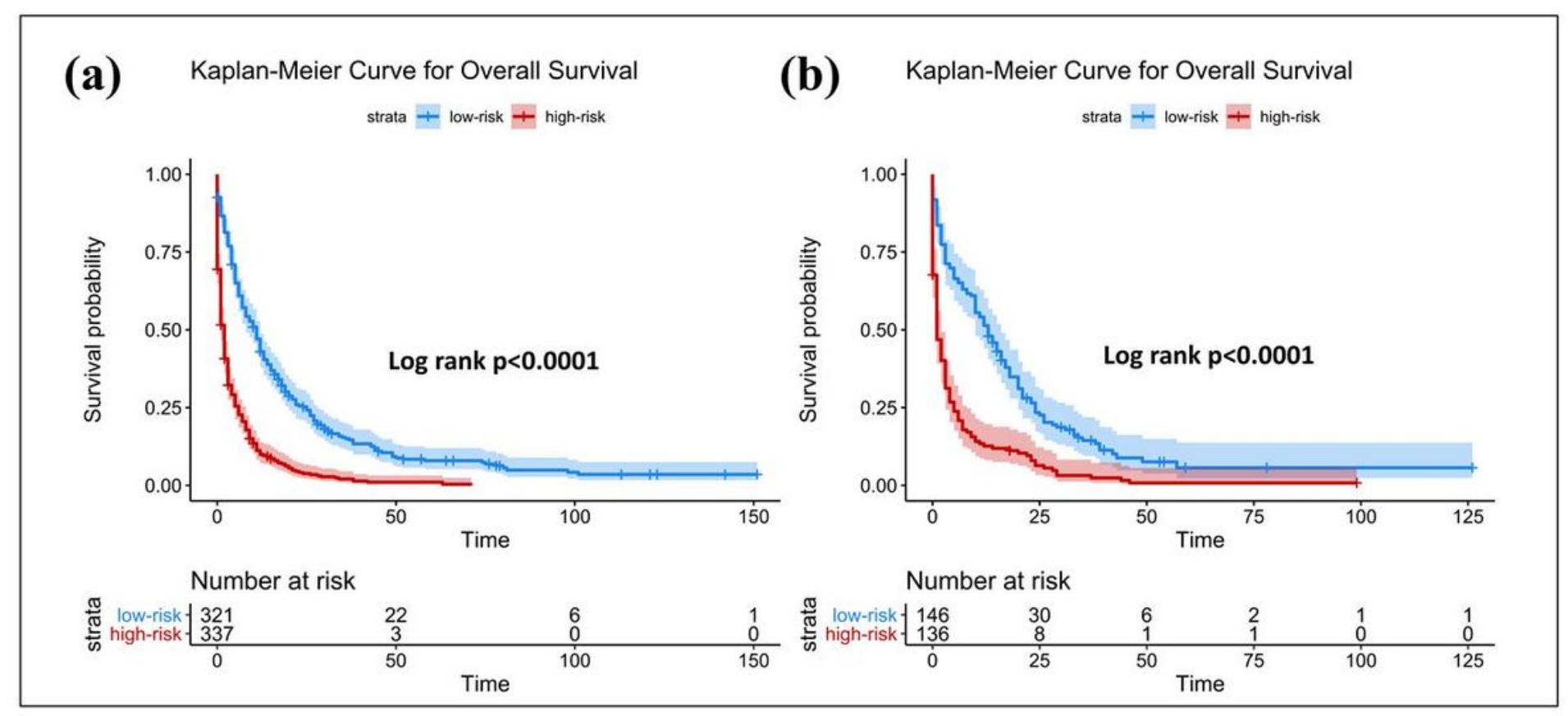

\section{Figure 5}

Kaplan-Meier survival curves according to the risk levels of nomogram-predicted survival probabilities: (a) the training set; (b) the validation set. 